\title{
Phakic Iris-Fixated Intraocular Lens Placement in the Anterior Chamber: Effects on Aqueous Flow
}

\author{
Rodolfo Repetto, ${ }^{1}$ Jan O. Pralits, ${ }^{1}$ Jennifer H. Siggers, ${ }^{2}$ and Paolo Soleri ${ }^{3}$ \\ ${ }^{1}$ Department of Civil, Chemical and Environmental Engineering, University of Genoa, Genoa, Italy \\ ${ }^{2}$ Department of Bioengineering, Imperial College London, London, United Kingdom \\ ${ }^{3}$ Ophtec BV, Groningen, The Netherlands
}

Correspondence: Jennifer H. Siggers, Department of Bioengineering, Imperial College London, London SW7 2AZ, UK;

j.siggers@imperial.ac.uk.

Submitted: November 21, 2014

Accepted: March 25, 2015

Citation: Repetto R, Pralits JO, Siggers JH, Soleri P. Phakic iris-fixated intraocular lens placement in the anterior chamber: effects on aqueous flow. Invest Ophthalmol Vis Sci. 2015;56:3061-3068. DOI:10.1167/ iovs.14-16118
Purpose. Phakic intraocular lenses (pIOLs) are used for correcting vision; in this paper we investigate the fluid dynamical effects of an iris-fixated lens in the anterior chamber. In particular, we focus on changes in the wall shear stress (WSS) on the cornea and iris, which could be responsible for endothelial and pigment cell loss, respectively, and also on the possible increase of the intraocular pressure, which is known to correlate with the incidence of secondary glaucoma.

Methods. We use a mathematical model to study fluid flow in the anterior chamber in the presence of a pIOL. The governing equations are solved numerically using the open source software OpenFOAM. We use an idealized standard geometry for the anterior chamber and a realistic geometric description of the pIOL.

Results. We consider separately the main mechanisms that produce fluid flow in the anterior chamber. The numerical simulations allow us to obtain a detailed description of the velocity and pressure distribution in the anterior chamber, and indicated that implantation of the pIOL significantly modifies the fluid dynamics in the anterior chamber. However, lens implantation has negligible influence on the intraocular pressure and does not produce a significant increase of the shear stress on the cornea, while the shear stress on the iris, although increased, is not enough to cause detachment of cells.

Conclusions. We conclude that alterations in the fluid dynamics in the anterior chamber as a result of lens implantation are unlikely to be the cause of medical complications associated with its use.

Keywords: aqueous flow, shear stress, intraocular pressure

\begin{abstract}
Surgical interventions for refractive error correction have $\checkmark$ become a viable alternative to the wearing of spectacles and contact lenses. The most commonly adopted option is laser surgical remodeling of the cornea; however, eyes with insufficient corneal thickness or very high refraction errors are unsuitable for this surgery, but may be treated by the implantation of an artificial intraocular lens.

Phakic intraocular lenses (pIOLs) are a type of lens that is inserted in the eye without removing or altering the natural crystalline lens. There are three main types: sulcus-supported lenses are placed in the posterior chamber, while anglesupported lenses and iris-fixated lenses are placed in the anterior chamber. In the present paper we focus on iris-fixated lenses, originally developed by Worst ${ }^{1}$ and Fechner et al., ${ }^{2}$ which, as their name suggests, are held in place relative to the iris by haptics with claws that attach to the iris. These devices are marketed by Ophtec BV (Groningen, The Netherlands).

Phakic IOLs are associated with some possible complications, which, according to Kwitko and Stolz, ${ }^{3}$ include chronic subclinical inflammation, corneal endothelial cell loss, cataract formation, secondary glaucoma, iris atrophy, and dislocation. In this paper we focus on three risks associated with pIOLs that could have mechanical underpinnings:
\end{abstract}

- Possible increase of pressure in the posterior chamber: The presence of the pIOL might increase the resistance to flow from the posterior to the anterior chambers, increasing the pressure in the posterior chamber that is required to maintain the flow. This risk is commonly mitigated by introducing a laser iridotomy in the iris, and it would be useful to know which patients would benefit from this treatment.

- Reduction of endothelial cell density on the posterior surface of the cornea: This could occur due to excessive wall shear stress arising from the altered flow patterns in the anterior chamber. ${ }^{4}$

- Loss of pigment cells from the iris: This could also be due to excessive wall shear stress due to changes in the fluid dynamics of the anterior chamber.

- Secondary glaucoma: This is possibly caused by increasing the resistance to the flow of aqueous humor, either within the anterior chamber or at the outflow.

Our aim was to investigate mechanical changes resulting from pIOL placement that could lead to increased health risk. This problem has been considered in the case of posterior chamber pIOL, the use of which has been growing in recent years. ${ }^{5}$ However, to our knowledge the only work in which this problem was considered was done by Niazi et al., ${ }^{6}$ who proposed a numerical model to study how aqueous flow induced by temperature differences between the front and the back parts of the anterior chamber is modified after implanta- 
TABLE. Parameter Values Used for the Simulations

\begin{tabular}{|c|c|c|}
\hline Quantity & Value & References \\
\hline \multicolumn{3}{|l|}{ Aqueous properties } \\
\hline Density $\rho$ & $1000 \mathrm{~kg} / \mathrm{m}^{3}$ & \\
\hline Volumetric flux $Q$ & $3 \mu \mathrm{L} / \mathrm{min}=5 \cdot 10^{-11} \mathrm{~m}^{3} / \mathrm{s}$ & 9,10 \\
\hline Kinematic viscosity $v$ & $7.5 \cdot 10^{-7} \mathrm{~m}^{2} / \mathrm{s}$ & 11 \\
\hline Specific heat at constant pressure $c_{p}$ & $4.178 \cdot 10^{3} \mathrm{~J} \mathrm{~kg}^{-1} \mathrm{~K}^{-1}$ & 12 \\
\hline Thermal conductivity $k$ & $0.578 \mathrm{~W} \mathrm{~m}^{-1} \mathrm{~K}^{-1}$ & 13 \\
\hline Thermal expansion coefficient $\alpha$ & $3 \cdot 10^{-4} \mathrm{~K}^{-1}$ & 12 \\
\hline \multicolumn{3}{|l|}{ Geometric characteristics of the domain } \\
\hline Diameter of the anterior chamber $D_{C}$ & $13 \mathrm{~mm}$ & \\
\hline Maximum height of chamber $b_{C}$ & $2.63 \mathrm{~mm}$ & ISO-11979-3 $3^{14}$ \\
\hline Minimum radius of curvature of the posterior cornea $R_{C}$ & $6.8 \mathrm{~mm}$ & ISO-11979-3 $3^{14}$ \\
\hline Radius of curvature of the natural lens $R_{L}$ & $10 \mathrm{~mm}$ & \\
\hline Height of the iris-lens channel & $0.1 \mathrm{~mm}$ & \\
\hline Angle between cornea and iris & $30^{\circ}$ & \\
\hline Overall diameter of the pIOL & $8.5 \mathrm{~mm}$ & \\
\hline Body diameter of the pIOL & $6 \mathrm{~mm}$ & \\
\hline Claw height on the pIOL & $0.26 \mathrm{~mm}$ & \\
\hline
\end{tabular}

tion of an iris-fixated pIOL. In the current paper we also present the results of a numerical model that allows us to predict the change in the pressure distribution due to the lens, as well as the changes in the shear stress on the surfaces of the iris and cornea. We extend the work by Niazi et $a{ }^{6}{ }^{6}$ by considering various mechanisms that can produce fluid flow in the anterior chamber and, specifically, focusing on the flow due to the production and drainage of aqueous humor, the flow due to miosis of the pupil, the buoyancy-driven flow that arises from density variations due to the temperature gradient across the anterior chamber, and also the changes that are produced due to the flow generated during saccades of the eye. In each case we calculate the distributions of pressure and wall shear stress (WSS) to estimate whether there is cause for concern regarding the use of pIOLs.

\section{Materials AND Methods}

\section{Description of the Model}

In this paper we develop a mathematical model to study the flow of aqueous humor in the anterior chamber with a pIOL. The main mechanisms that generate flow of the aqueous humor are (1) flow due to aqueous production and drainage, (2) flow due to miosis of the pupil, (3) buoyancy-driven flow due to temperature differences between the anterior and the posterior regions of the anterior chamber, and (4) motion induced by saccades of the eye.

The aqueous humor is treated as an incompressible Newtonian viscous fluid governed by the momentum (Navier-Stokes) and continuity equations, which read

$$
\begin{aligned}
\frac{\partial u}{\partial t}+(u \cdot \nabla) u+\frac{1}{\rho} \nabla p-v \nabla^{2} u-g & =0, \\
\nabla \cdot u & =0,
\end{aligned}
$$

where $u$ denotes the velocity vector, $p$ is pressure, $t$ time, $\rho$ fluid density, $v$ the fluid kinematic viscosity, and $g$ the gravitational acceleration, with magnitude $g=|g|$. When we study the flow induced by rotations of the eye, the above equations are solved on a moving domain, and therefore have to be suitably modified (see the OpenFOAM website for further details: http://www. openfoam.org/features/ [in the public domain]).
In the case of buoyancy-driven flow, the above equations are coupled to the energy equation

$$
\frac{\partial T}{\partial t}+(u \cdot \nabla) T-\frac{k}{\rho c_{p}} \nabla^{2} T=0
$$

where $T$ is temperature, $k$ is the thermal conductivity of the fluid, and $c_{p}$ is the specific heat at constant pressure. In Equation 2 we have neglected the heat production due to mechanical energy dissipation, which is negligible in the present context. We adopt the Boussinesq approximation (see, for instance, Drazin and $\operatorname{Reid}^{7}$ ), which is known to be very accurate for liquids undergoing small variations in temperature. In this approximation we neglect variations in the fluid density except in the gravitational forcing term, and we neglect variations in the kinematic viscosity $v$. In this term we assume that the aqueous humor density $\rho$ has a small linear dependency on temperature and is independent of pressure, leading to

$$
\rho=\rho_{0}\left[1-\alpha\left(T-T_{0}\right)\right],
$$

where $\rho_{0}$ is the fluid density at a reference temperature $T_{0}$, and $\alpha$ is the linear thermal expansion coefficient of the fluid.

We solve Equations 1 and 2 numerically using the open source software OpenFOAM ${ }^{8}$ which is based on the finite volume method. All meshes are generated using the snappyHexMesh tool by OpenFOAM, which produces unstructured meshes consisting of tetragonal and hexahedral volumes. We adopt meshes consisting on average of 1 to 2 million volumes, except when analyzing the lens including the haptics for which the mesh has 2.7 million volumes, and run the code in parallel on a 32 processor computer (32 processor work station from the E4 Company, Scandiano, Italy). For all simulations, careful mesh-independence tests have been carried out. In the Table, we report the parameter values used for the simulations.

One parameter that we will discuss in the course of the paper is the WSS, an excessive value of which can be a possible cause of cell detachment from the cornea and iris. We compute this quantity with the wallShearStress utility by OpenFOAM. We note that this tool computes the magnitude of the viscous traction at the wall, including its normal component. However, it can be shown that, if the fluid is incompressible, the normal component of the viscous traction at a rigid wall is zero. Thus, leaving aside numerical errors, the magnitude of the viscous traction is indeed equal to the WSS in all cases considered in the present work. 


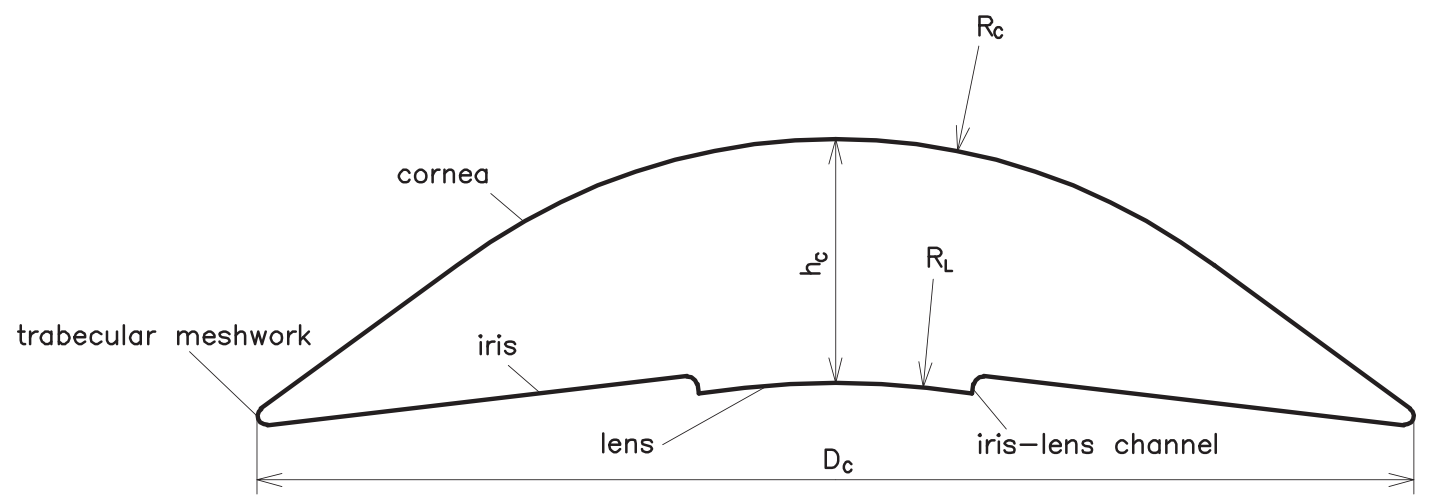

Figure 1. Cross section of the idealized anterior chamber used in the simulations.

\section{Model Geometry}

We consider the geometry shown in Figure 1, which represents an idealization of the real geometry. Specifically, we assume that the chamber is axisymmetric and we model the cross section of the cornea as a central arc of a circle with straight lines representing the outer regions, the cross section of the lens as a circular arc, and the cross section of the iris as a straight line. We smooth the joins between the different tissues. The corresponding parameter values are given in the Table, and a file is provided as additional material (interested readers can contact the authors for the geometrical detail of the anterior chamber).

For the model of the pIOL we use the geometry of the Artiflex lens, provided by the manufacturer (Ophtec BV). A view of the geometry of the pIOL is shown in Figure 2a. The pIOL consists of a body (lens) and two haptics (claws). In order to fixate the pIOL to the iris, the haptics are used to grab a piece of iris tissue. The pIOL is placed in the anterior chamber, as shown schematically in Figure $2 b$. In most of the simulations in this paper we neglect the haptics, which has the advantages that (1) we can easily change the location of the pIOL to investigate the effect of device positioning, and (2) we may use smaller meshes, which significantly reduces the computational time, which is especially important for the unsteady simulations. To check the validity of this assumption we also ran simulations with the haptics present, and we invariably found that their effect on the flow is very small. Furthermore, for all simulations considered we compared the results with benchmark simulations without the pIOL.

We consider four different sets of simulations, each with a slightly different setup, which are designed to test the effect of a particular flow mechanism: production and drainage of the aqueous humor, miosis, buoyancy-driven flow, and saccades of the eye. The adaptations to the model are described in the following sections.

\section{Flow Induced by Aqueous Production and Drainage}

Aqueous humor is produced by the ciliary body, and then flows through the posterior chamber, the pupil, and the anterior chamber; from there it is drained into the trabecular meshwork. The inlet is at the iris-lens channel, and we assume a radially inward flow $Q$ that is uniformly distributed over this surface. The outlet is at the trabecular meshwork, where we impose zero pressure. This means that the computed values of the pressure actually represent pressure differences with respect to the outlet. The pressure is, therefore, defined up to a constant that does not affect the results. All the other surfaces are rigid walls at which we impose a no-slip boundary condition. We obtain the solution using the simpleFoam solver, which uses a semi-implicit method to solve the Navier-Stokes equations iteratively.

\section{Flow Induced During Miosis}

During pupil contraction (miosis), a flow from the posterior to the anterior chamber of the eye is generated, which is more intense than the bulk flow due to production/drainage of aqueous described in the previous section, although it lasts only a short time, typically less than 1 second. Yamamoto et al. ${ }^{15}$ used PIV (particle image velocimetry) to measure the flow of aqueous humor through a laser iridotomy hole during miosis in rabbits. These results were used by Yamamoto et al. ${ }^{16}$ to prescribe the inlet velocity of an iridotomy jet in their numerical model of flow in the anterior chamber, which they assumed to be the first quarter-period of a cosine curve. They used a hole of diameter $d_{L I}=0.56 \mathrm{~mm}$ for which the measured maximum velocity was $u_{\max }=9.39 \mathrm{~mm} / \mathrm{s}$, and took the duration to be the average duration of miosis in humans after light stimulation, ${ }^{17}$ giving $T_{j e t}=0.66$ seconds.

If we assume that, during miosis in an eye with an iridotomy, the volumetric flux through the iris-lens channel is negligible compared to that through the iridotomy hole, it would suggest that the estimates of volumetric flux through the iridotomy in the experiments of Yamamoto et al. ${ }^{15}$ could be similar to the volumetric flux through the iris-lens channel in the natural eye. We therefore adopt the formula used by (a)

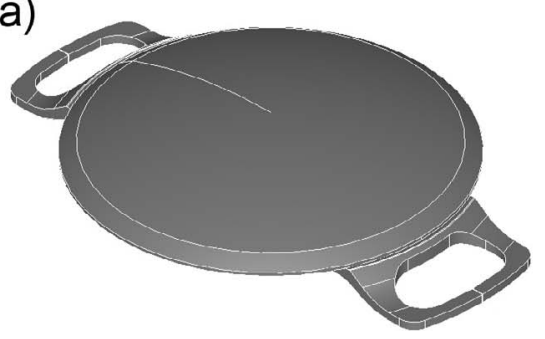

(b)

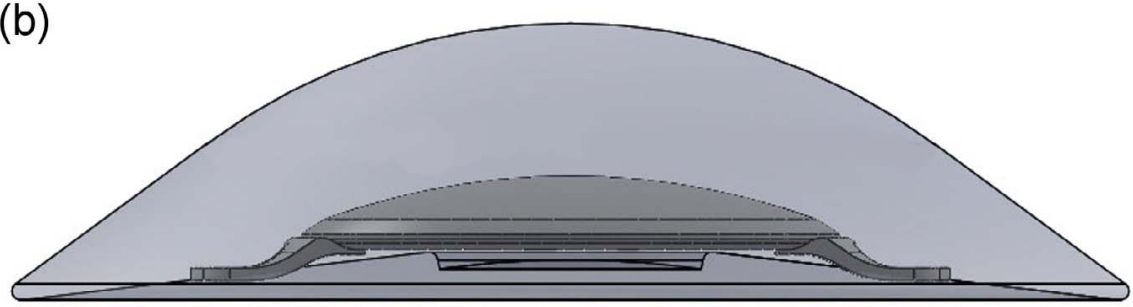

Figure 2. (a) Geometry of the pIOL consisting of a lens and two haptics with claws that allow the lens to be attached to the iris, (b) pIOL placed in the anterior chamber. 

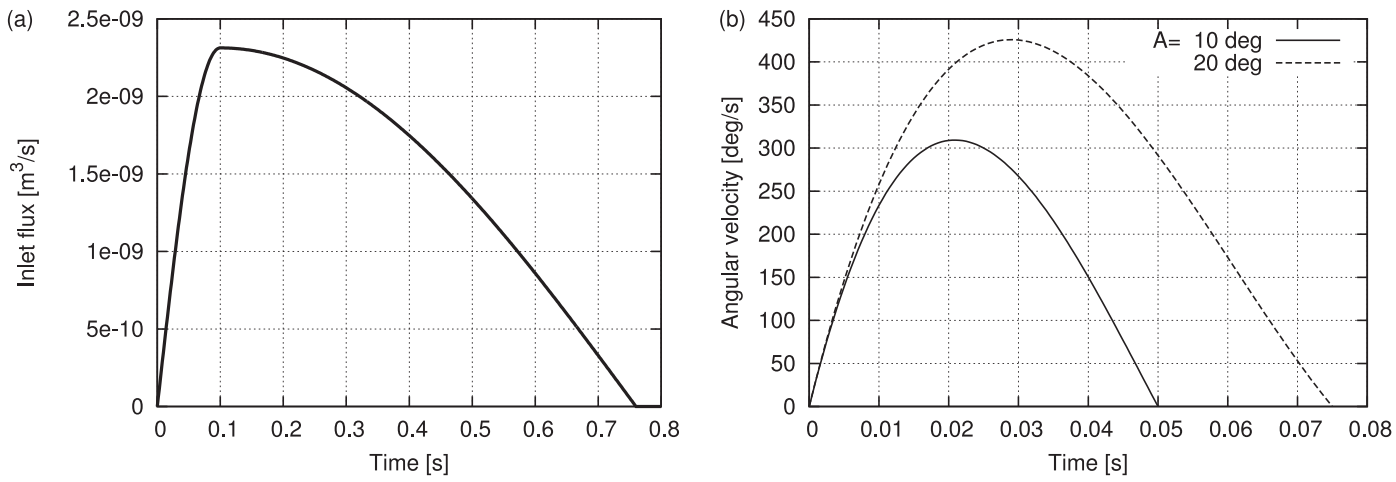

Figure 3. (a) Flux from the posterior to the anterior chamber of the eye generated during miosis. The intensity of the flux is based on the measurements by Yamamoto et al. ${ }^{16}$ on rabbit eyes. (b) Angular velocity of the eye globe versus time during saccadic rotations for two different saccadic rotations with amplitudes $10^{\circ}$ and $20^{\circ}$.

Yamamoto et al. ${ }^{16}$ and impose the following time-dependent total volumetric flux through the iris-lens channel in our simulations of miosis in the natural eye:

$$
Q(t)=\pi \frac{d_{L I}^{2}}{4} u_{\max } \cos \left(\frac{\pi\left(t-t_{\text {init }}\right)}{2 T_{\text {jet }}}\right),
$$

for $t_{\text {init }} \leq t \leq t_{\text {init }}+T_{\text {jet }}$, where $t$ is time. To avoid numerical artifacts, we also added a ramp during $0 \leq t \leq t_{\text {init }}=0.1$ seconds during which the velocity grows sinusoidally from 0 to $u_{\text {max }}$; see Figure $3 a$.

We assume that this flux is uniformly distributed over the surface of the iris-lens channel, apply zero pressure at the trabecular meshwork and a no-slip velocity on all other surfaces. The use of the zero pressure condition at the outlet section requires some comments. The flow from the posterior to the anterior chamber generated during miosis exits the domain through the trabecular meshwork and will produce a variation in time of the outlet pressure. This can be accounted for adopting a boundary condition that relates the pressure to the flux, as has been proposed by Heys et al. ${ }^{18}$ In the present case, owing to the fact that we consider an incompressible fluid and a noncompliant domain, the outlet flux is instantaneously equal to the inlet flux, as a consequence of mass conservation. This implies that the use of the boundary condition proposed by Heys et al. ${ }^{18}$ would produce an unsteady pressure variation at the outlet section, which can be directly related, through an outflow resistance, to the inlet flux. Such pressure change, being uniform across the domain, does not modify the flow. In other words, using the zero pressure outlet condition implies that the computed pressure represents the instantaneous difference between the local and the outlet pressure, which is in any case the pressure difference of interest to us in this study.

We use the icoFoam solver for unsteady incompressible laminar flow to find the solution.

\section{Buoyancy-Driven Flow}

It is well known that, since the posterior surface of the cornea is typically cooler than the iris and lens, the fluid in the front part of the anterior chamber is denser than that toward the posterior, and so the fluid at the anterior tends to sink, while that at the posterior rises. This flow has been studied analytically, numerically, and experimentally (e.g., Refs. 15, 19, 20). Under normal conditions (i.e., an upright subject with open eyelids in an atmosphere that is several degrees below body temperature) this buoyancy-driven flow is significantly more intense than the flow due to production/drainage of the aqueous, and therefore we neglect inflow and outflow in these simulations.

The material often used in manufacturing iris-fixated pIOLs has a relatively low thermal conductivity, and so we neglect heat transport in the device. Niazi et al. ${ }^{6}$ showed that the typical velocities are significantly reduced for greater values of the conductivity of the device, and thus the WSS also decreases with increasing thermal conductivity. Since our aim is to determine the risk of excess WSS on the iris and cornea, we expect the present study to provide an estimate of the worstcase scenario regarding the WSS.

We simulate the governing equations, together with no-slip conditions on all boundaries, and a prescribed temperature of $34^{\circ} \mathrm{C}$ on the cornea and $37^{\circ} \mathrm{C}$ on all other surfaces. We use the simpleBuoyantBoussinesqFoam solver, which uses a semiimplicit method to solve the equations iteratively.

\section{Flow Induced by Saccades of the Eye}

Finally, we consider the flow generated in the anterior chamber by rotations of the eye bulb. We model isolated rotations using the analytical relationship proposed by Repetto et al., ${ }^{21}$ which provides the angular velocity of the eye as a function of time; see the examples in Figure 3b. As for the buoyancy-driven flow described in the previous section, since the fluid velocities induced by the production and drainage of aqueous humor are expected to be significantly smaller than those induced by saccades, we neglect production and drainage and impose a no-slip velocity on all boundaries. We use the unsteady solver pimpleDyMFoam to simulate the Navier-Stokes and continuity equations on a moving grid.

\section{Results}

\section{Flow Induced by Aqueous Production and Drainage}

For this flow, unless the haptics on the pIOL are included in the simulation, both the geometry and the boundary conditions are axisymmetric, and therefore the resulting flow is also axisymmetric. The resulting pressure and velocity distribution with pIOL are given in Figure 4, showing that the pressure is highest in the region posterior to the pIOL, although this difference is too small to be clinically relevant. The velocity magnitude has peaks at the inlet and outlet and also in the region between the pIOL and the iris. For brevity we have omitted the corresponding results in the natural eye, but in that case the pressure variation is approximately half that in Figure $4 \mathrm{a}$ and peaks in the velocity are obtained only at the inlet and outlet. 


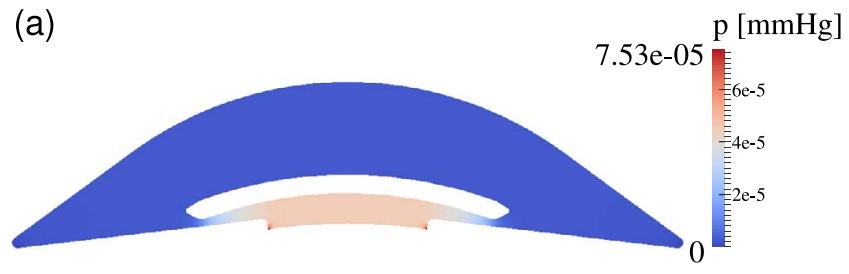

(b)

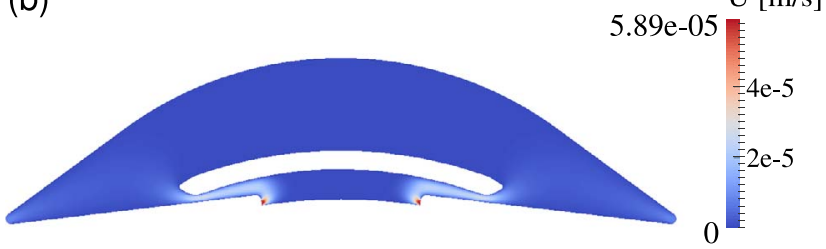

FiguRe 4. Flow due to production/drainage of aqueous humor with the device present: (a) excess pressure above intraocular pressure, (b) velocity magnitude.

In the presence of the haptics, flow characteristics are very similar. However, the haptics impose a preferential direction to the aqueous velocity that breaks the axial symmetry of the flow. Specifically, they partially obstruct the flow, which is thus forced toward the sides of the lens. We note, moreover, that in the presence of the haptics, specific regions characterized by low values of the velocity (at the front and rear sides of the haptics) occur. In these regions there may be an increased risk of particle deposition, such as pigmentary cells, leukocytes, and erythrocytes, as suggested by Kumar et al. ${ }^{22}$ A study of this process is outside of the scope of the present paper but might deserve future attention.

We also investigate the role of the positioning of the pIOL, and, neglecting the haptics, we progressively displace the lens posteriorly, recording the pressure drop at each position, which grows nonlinearly as the pIOL approaches the iris; see the solid circles in Figure 5. We also show the pressure drop corresponding to the simulation including the haptics (empty circle), indicating that the haptics have a negligible effect on the pressure drop. Finally, we report in the figure the pressure drop in the absence of the pIOL, which is significantly lower, although it must be emphasized that even the largest pressure drop with the pIOL close to the iris $\left(2.55 \times 10^{-4} \mathrm{~mm} \mathrm{Hg}\right)$ is too small to be clinically relevant.

To investigate an even closer positioning of the pIOL to the iris, we cannot use the numerical simulations, as the gap is too small to be sufficiently resolved for numerical accuracy. However, using lubrication theory we can find an analytical estimate of the pressure drop across a small gap as

$$
\Delta p=\frac{6 \mu Q}{\pi} \int_{r_{1}}^{r_{2}} \frac{d r}{r b^{3}}
$$

where the gap has height $h(r)$ in the range $r_{1}<r<r_{2}$, with $r$ being the radial coordinate, $\mu$ is the shear viscosity of the aqueous humor, and $Q$ is the total volumetric flux through the anterior chamber. As a rough estimate, consider a device of radius $3 \mathrm{~mm}$ with a gap of uniform height and of width $1 \mathrm{~mm}$ at its outer edge. The formula above shows that, to obtain a pressure drop of $1 \mathrm{~mm} \mathrm{Hg}$ across the gap, the gap height needs to be approximately $6 \mu \mathrm{m}$, which is less than $5 \%$ of the normal distance between the pIOL and the iris. This estimate assumes that the lens remains axisymmetrically positioned, which is unlikely in the case of mispositioning; however, if the lens does not maintain close proximity to the iris all the way around its

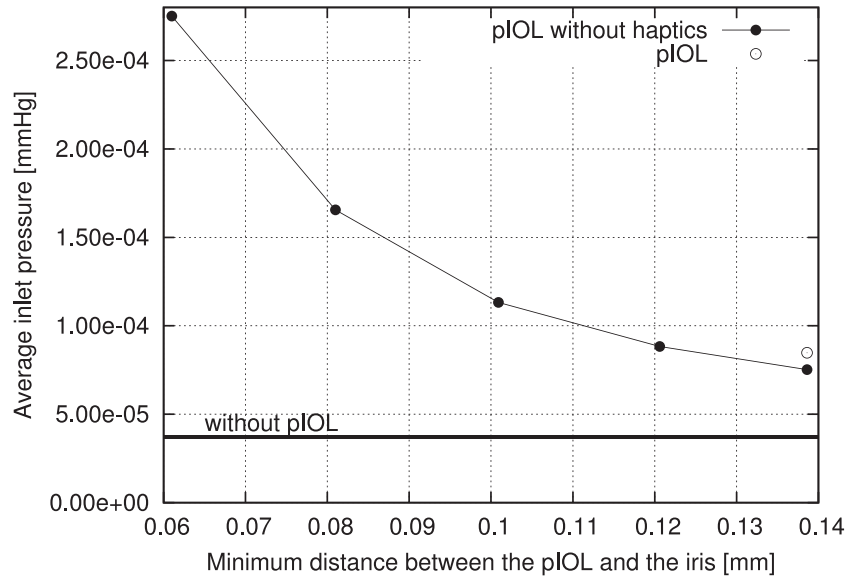

Figure 5. Average pressure drop between iris-lens channel and trabecular meshwork as a function of the minimum distance between the pIOL and the iris (solid circles). Also shown are the cases with no pIOL (borizontal line) and the case of a pIOL with haptics included in the simulated geometry (empty circle).

circumference, it would be likely to reduce the pressure drop further, because the fluid would have an alternative path to the trabecular meshwork. Furthermore, in reality the pIOLs in use do not have a flat region of width $1 \mathrm{~mm}$ near their edge, and the width of iris over which a gap of a few micrometers between iris and device could be maintained is much less than a millimeter. In summary, these estimates all indicate that it is highly unlikely that the increase in the pressure drop due to the flow needing to get through a narrow gap between the iris and device could ever be large enough so as to make a clinically significant difference.

The WSS on cornea and iris for this flow is always much smaller than physiologically relevant.

\section{Flow Induced During Miosis}

The results of the simulations of miosis are shown in Figure 6, in which we plot the pressure drop between the inlet and the outlet over time both with and without the pIOL in place. The pressure drop with the pIOL is approximately double that without the device (the same as for the flow due to the

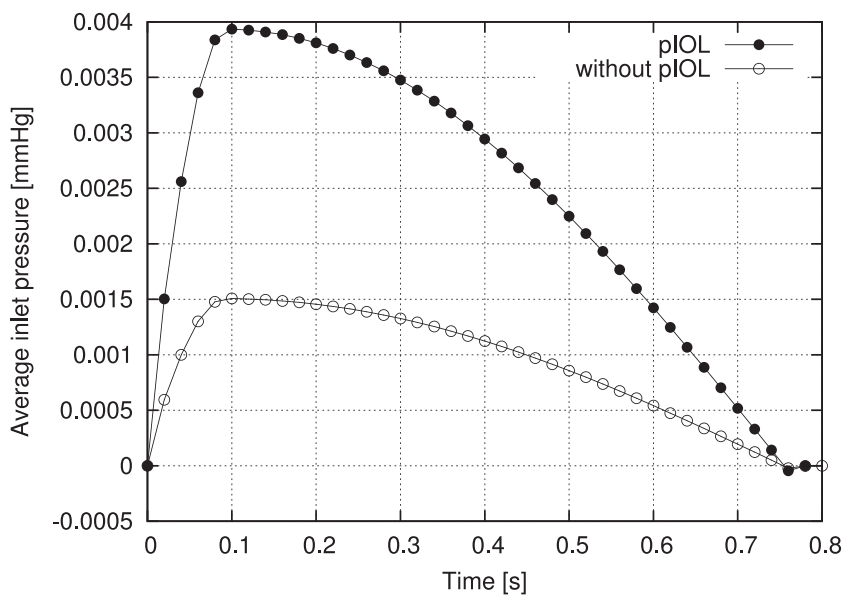

Figure 6. Spatially averaged pressure over the inlet as a function of time during miosis, in an eye both with the pIOL (solid circles) and without it (open circles). Note that the pressure in the figure has to be understood as the excess pressure (at each time) with respect to the outlet pressure. 
(a)

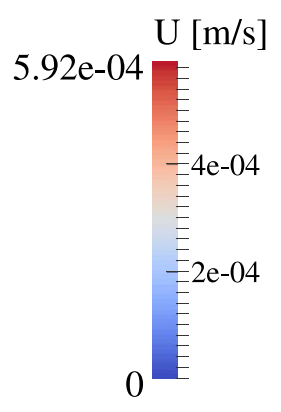

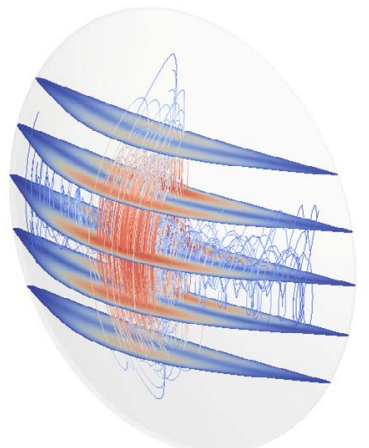

(b)

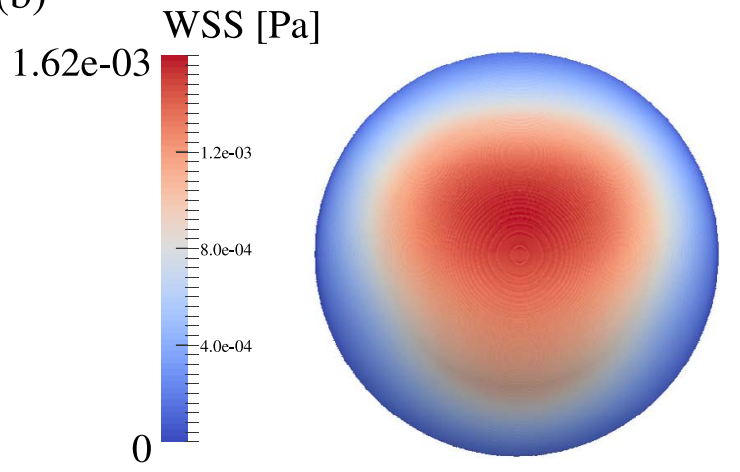

Figure 7. Buoyancy-driven flow in the absence of the pIOL. In this and subsequent figures, gravity acts in the vertical direction. (a) Streamlines of the flow and the distribution of the velocity magnitude on selected horizontal planes. The velocity is directed downward in the anterior and upward in the posterior. (b) WSS on the cornea (the WSS on the iris is very similar).

production of aqueous humor), and, although the pressure drops are much larger in this case, they are still too small to be relevant clinically (thousands of times smaller than the intraocular pressure).

The WSS on the iris is also increased due to the pIOL during miosis, especially in the constricted region between the iris and the pIOL; and its maximum value over time and space equals $3.48 \times 10^{-2} \mathrm{~Pa}$ (pIOL with haptics) and $2.69 \times 10^{-2} \mathrm{~Pa}$ (pIOL with no haptics), whereas it is almost zero with no pIOL.

In all cases considered, the WSS on the cornea is negligibly small.

\section{Buoyancy-Driven Flow}

In this section we will first present results in the natural eye, followed by the results with the pIOL in place. We show the streamlines and the velocity magnitude in Figure $7 \mathrm{a}$; and as predicted by previous investigations, ${ }^{6,19}$ the flow is approximately confined to two-dimensional vertical planes. The maximum velocity is higher by approximately two orders of magnitude than that of the flow due to the production of aqueous humor. These results agree very well with the theoretical predictions by Canning et al. ${ }^{19}$ and also justify the fact that we have neglected the flow due to the production of aqueous humor. In Figure $7 \mathrm{~b}$ we show the corresponding corneal WSS stress, which attains a maximum value of $1.64 \times$ $10^{-3} \mathrm{~Pa}$ in the center of the cornea.

The pIOL significantly modifies this flow, which can be seen by comparing Figure 8 with Figure $7 \mathrm{a}$. In particular, the flow is now three-dimensional, and particles anterior to the pIOL tend to move down and then move laterally out to the sides before moving back up around the sides of the device. There is very
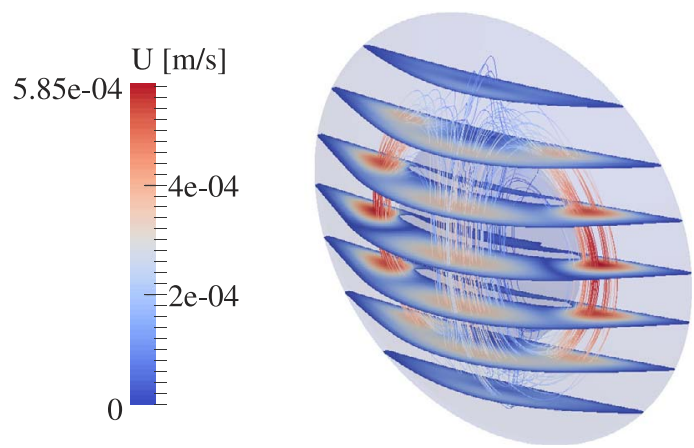

FIGURE 8. Buoyancy-driven flow, as for Figure $7 \mathrm{a}$, but with the pIOL in place. little flow posterior to the lens. The streamlines shown in the figures are in very good qualitative agreement with those computed by Niazi et al. ${ }^{6}$ Even though the flow structure is significantly modified by the presence of the pIOL, the maximum absolute velocity is very similar to that without the pIOL. We note, however, that Niazi et al. ${ }^{6}$ showed that if a pIOL with a high thermal diffusion coefficient is used, aqueous humor velocity can be significantly decreased.

We show the corresponding WSS on the cornea and iris in Figure 9, and this also indicates significant changes due to the pIOL. The maximum WSS stress on the cornea is slightly smaller than it is in the absence of the lens, while the WSS on the iris peaks both at the sides of the iris, where there is a relatively intense upward flow, and also at the top and bottom of the narrow gap between the iris and the pIOL. The maximum WSS on the iris is approximately twice as large as that on the cornea.

Since the corneal WSS is of clinical importance, we plot its dependence upon the position of the PIOL in Figure 10. The maximum WSS is greater the greater the distance between the pIOL and the iris, and we can also see that the presence of the haptics makes little difference to the maximum WSS. The maximum WSS on the cornea with no pIOL is also shown, which indicates that the pIOL actually reduces the WSS. This is to be expected because the presence of the pIOL increases the resistance to the buoyancy-driven flow by reducing the space available for it to take place. Since the no-slip boundary condition must be satisfied on all surfaces, even a zerothickness device changes the flow pattern (in addition to any change caused by the different temperature profile).

\section{Flow Induced by Saccades of the Eye}

Inclusion of the haptics in a problem with a moving grid would come at significant cost in terms of computational time, and so we neglect them in this section. In Figures $11 \mathrm{a}$ and $11 \mathrm{~b}$ we plot the time evolution of the spatial maximum of the WSS on the cornea (Fig. 11a) and iris (Fig. 11b) in the absence (open circles) and presence (solid circles) of the pIOL. The time evolution of the WSS on the cornea with no pIOL agrees closely (both qualitatively and quantitatively) with that obtained numerically by Abouali et al. ${ }^{23}$ (see fig. 4 of that paper, red line). We also note that the WSS distribution on the cornea is not significantly modified by the presence of the pIOL, and it is in agreement with the distribution shown by Abouali et al. ${ }^{23}$ (see fig. 3 of that paper).

The time evolution of the corneal WSS with the PIOL is qualitatively the same as that without, although the maximum over time is slightly greater without the pIOL. Furthermore, the 
(a)

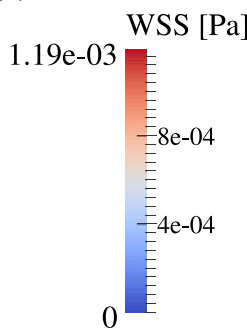

(b)

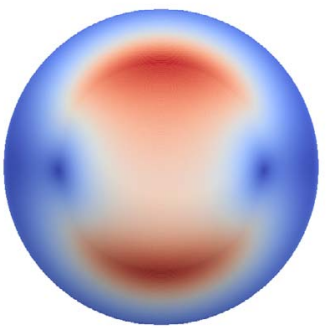

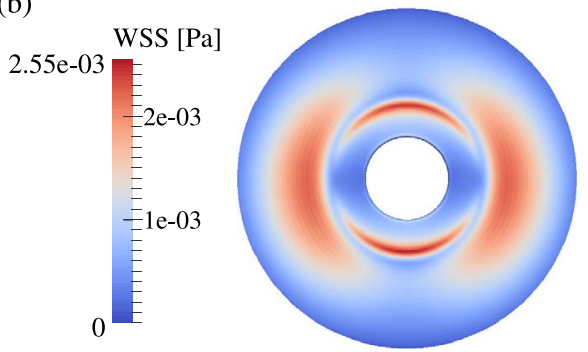

Figure 9. Buoyancy-driven flow in the presence of the pIOL. (a) Magnitude of WSS on the cornea; (b) WSS on the iris.

value of the maximum WSS is much greater, by a factor of at least six, than that for buoyancy-driven flow.

On the other hand, pIOL implantation produces an increase of the maximum WSS on the iris by a factor of approximately 1.5 with respect to the case of no pIOL.

\section{Discussion}

In this paper we have performed numerical simulations to investigate how iris-fixated pIOL placement modifies the fluid mechanics of the anterior chamber. A similar problem was considered for the case of posterior chamber pIOLs by Kawamorita et al. ${ }^{5}$ There is a strong clinical interest in this, because iris-fixated pIOL implantation is associated with increased risk of secondary glaucoma due to a possible increase in the pressure in the posterior chamber and also with increased rate of loss of endothelial corneal cells and pigment cells from the iris 3 ; and these complications could arise as a result of changes in the fluid flow following pIOL placement. The risk of secondary glaucoma is typically mitigated by introducing an iridotomy into the iris; however, the risk of secondary glaucoma with no iridotomy is currently unknown. The only existing work in which the effect of irisfixated pIOLs on the fluid dynamics of the aqueous humor is considered is that of Niazi et al., ${ }^{6}$ who investigated changes in the thermally driven flow. We have extended their work by also accounting for various other mechanisms of fluid motion in the anterior chamber. Since in vivo measurements of aqueous flow would be extremely challenging (and are not available at present), a numerical approach seems to be the best option.

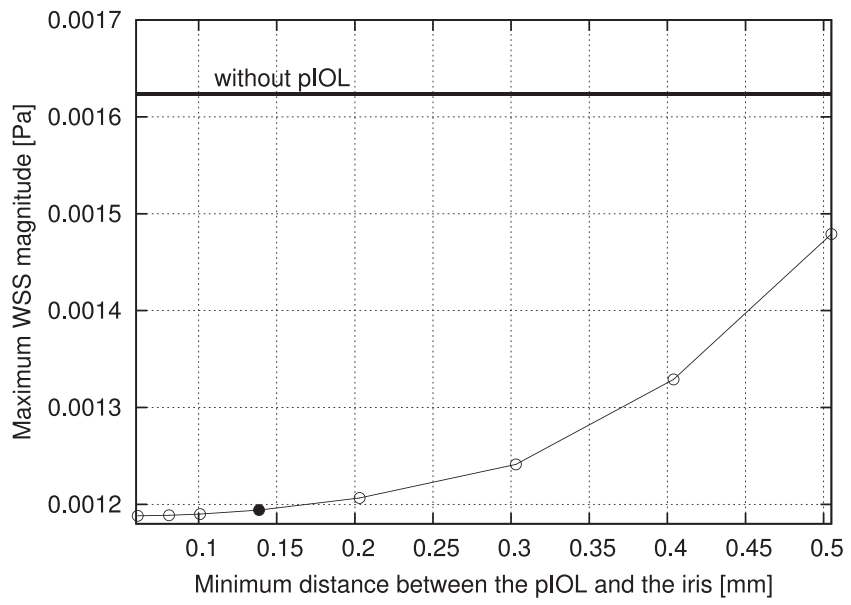

FIGURE 10. Spatial maximum of the WSS on the cornea as a function of the distance between the pIOL and the iris, neglecting the haptics (open circles). The maximum WSS with no pIOL is shown by the borizontal line, while the maximum WSS with the PIOL in its normal position and including the haptics is shown by the solid circle.
Owing to the lack of pressure and aqueous velocity measurements in vivo, our results cannot be validated against experimental data. However, owing to the laminar nature of the flows considered (numerical simulations of laminar flows are notoriously much easier than of turbulent flows), we believe our numerical results to be quite reliable.

We considered the four main mechanisms that generate flow of the aqueous humor in turn: production and drainage of aqueous humor, miosis, buoyancy-driven flow, and saccadedriven flow. We looked specifically at the pressure drop between the inlet (iris-lens channel) and outlet (trabecular meshwork), and in each case compared our results to a similar simulation with no pIOL.

We neglected density differences between the device and the aqueous humor and modeled the anterior chamber as an axisymmetric domain that closely matches the real shape, and the model pIOL geometry was based on the geometry of the Artiflex lens, which was provided by Ophtec BV. The pIOL has a body (the actual lens) and haptics, and we performed simulations both with and without the haptics in the model; disregarding the haptics did not significantly affect the flow, greatly reduced the computational time, and also allowed the pIOL to be easily moved around the domain. Our simulations show how the fluid mechanics of the anterior chamber is modified by implantation of a pIOL, and the main conclusions of the work are that implantation of a pIOL has the following effects:

1. If the lens is properly placed, there is a negligible influence on the pressure in the posterior chamber.

2. There is no significant increase of the WSS on the cornea.

3. The WSS on the iris is significantly greater than in the case with no pIOL, but the increase is not likely to be sufficiently great so as to give a risk of cell detachment.

Kaji et al. ${ }^{24}$ performed experiments on porcine corneal endothelial cells that were plated onto glass slides, and found that significant detachment was observed for shear stresses in excess of $0.03 \mathrm{~Pa}$ if the cells had had 1 hour of adhesion, rising to $0.1 \mathrm{~Pa}$ for 3 hours of adhesion. Thus in vivo, the critical WSS at which cells start to detach from the cornea is likely to be in excess of $0.1 \mathrm{~Pa}$. Our model predicts that the actual values are significantly smaller than this, and so it is unlikely that WSS is responsible for detaching the cells from the cornea. Although we do not have corresponding data for the iris, the result is likely to be similar for that too.

Thus the mechanism behind the observed detachment of corneal endothelial cells remains unknown. A possible explanation is that the patient could rub her/his eyes, bringing the cornea into contact with the pIOL, leading to mechanical friction that causes the cells to detach. An alternative mechanism could be that there is insufficient delivery of oxygen and/or nutrients to the corneal endothelium. This could be especially true if there is no buoyancy-driven flow, for 

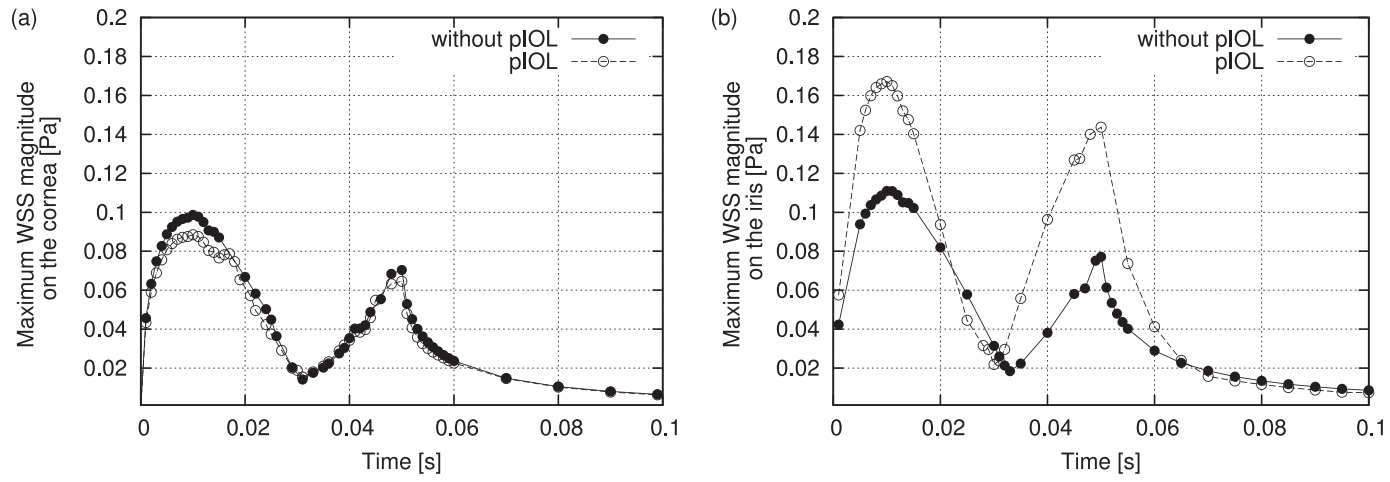

Figure 11. Time evolution of the spatial maximum of the WSS on (a) the cornea and (b) the iris in an eye performing a saccade of angle $10^{\circ}$.

example, during sleeping with eyelids closed; in this case the flow in the anterior chamber might be mainly driven by the production of aqueous humor. It has been suggested that rapid eye movements (REM) during sleeping may have the role of inducing some sloshing in the otherwise almost stagnant aqueous humor. It might be conceivable that, in spite of REMinduced aqueous motion, the presence of the pIOL, shielding the central cornea, could lead to a lower rate of oxygen and nutrient delivery there. This might in turn lead to the observed cell loss. We plan to test this hypothesis in future work by coupling the flow with the advection-diffusion equation for oxygen and nutrient transport.

\section{Acknowledgments}

Supported financially by Ophtec BV, Groningen, The Netherlands. Disclosure: R. Repetto, Ophtec BV (C); J.O. Pralits, None; J.H. Siggers, Ophtec BV (C); P. Soleri, Ophtec BV (E)

\section{References}

1. Worst JG. Iris claw lens. J Am Intraocul Implant Soc. 1980;6: 166-167.

2. Fechner P, Van der Heijde G, Worst J. Intraocular lens for the correction of myopia of the phakic eye [in German]. Klin Monbl Augenbeilkd. 1988;93:29-34.

3. Kwitko S, Stolz AP. Iris-claw (Artisan ${ }^{\circledR} /$ Artiflex $^{\circledR}$ ) phakic intraocular lenses for high myopia and high hyperopia. Expert Rev Ophthalmol. 2011;6:505-512.

4. Doors M, Berendschot TT, Webers CA, Nujits RM. Model to predict endothelial cell loss after iris-fixated phakic intraocular lens implantation. Invest Ophthalmol Vis Sci. 2010;51:811815.

5. Kawamorita T, Uozato H, Shimizu K. Fluid dynamics simulation of aqueous humour in a posterior-chamber phakic intraocular lens with a central perforation. Graefes Arch Clin Exp Ophthalmol. 2012;250:935-939.

6. Niazi M, Esteghamatian A, Abouali O, Ghaffariyeh A, Ahmadi G. Numerical study of natural convection in the anterior chamber of human eye with implanted intraocular lens. In: ASME 2012 Heat Transfer Summer Conference Collocated with the ASME 2012 Fluids Engineering Division Summer Meeting and the ASME 2012 10th International Conference on Nanochannels, Microchannels, and Minichannels. American Society of Mechanical Engineers; 2012:1007-1012.

7. Drazin PG, Reid WH. Hydrodynamic Stability. Cambridge, UK: Cambridge University Press; 1981:35-37.

8. OpenCFD, Ltd. ESI Group. OpenFOAM Web site. Available at http://openfoam.com. Accessed May 1, 2015.
9. Brubaker RF. Measurement of aqueous flow by fluorophotometry. In: The Glaucomas. St. Louis: Mosby; 1989.

10. Brubaker RF. Flow of aqueous humor in humans [the Friedenwald Lecture]. Invest Ophthalmol Vis Sci. 1991;32: 3145-3166.

11. Beswick JA, McCulloch C. Effect of hyaluronidase on the viscosity of the aqueous humour. Br J Ophthamol. 1956;40: 545-548.

12. Batchelor GK. An Introduction to Fluid Dynamics. Cambridge, UK: Cambridge University Press; 1967:596.

13. Poppendiek HF, Randall R, Breeden JA, Chambers JE, Murphy JR. Thermal conductivity measurements and predictions for biological fluids and tissues. Cryobiology. 1967;3:318-327.

14. Helmholtz H. Handbuch der physiologischen Optik. In: Karsten G, ed. Allgemeine Encyklopädie der Physik. Vol. 9. Leipzig: Voss; 1867.

15. Yamamoto $\mathrm{Y}$, Uno $\mathrm{T}$, Shisida $\mathrm{K}$, et al. Demonstration of aqueous streaming through a laser iridotomy window against the corneal endothelium. Arch Ophthalmol. 2006;124:387393.

16. Yamamoto Y, Uno T, Joko T, Shiraishi A, Ohashi Y. Effect of anterior chamber depth on shear stress exerted on corneal endothelial cells by altered aqueous flow after laser iridotomy. Invest Ophthalmol Vis Sci. 2010;51:1956-1964.

17. Onishi Y, Utsumi T, Hashimoto T, Namba K. Pupillary dynamics under open-loop photic stimulus, variations in age and sex. Folia Ophthalmol Jpn. 1979;30:921-926.

18. Heys JJ, Barocas VH, Taravella MJ. Modeling passive mechanical interaction between aqueous humor and iris. $J$ Biomech Eng. 2001;123:540-547.

19. Canning CR, Greaney MJ, Dewynne JN, Fitt A. Fluid flow in the anterior chamber of a human eye. IMA J Math ApplMed Biol. 2002;19:31-60.

20. Heys JJ, Barocas VH. A boussinesq model of natural convection in the human eye and formation of Krukenberg's spindle. Ann Biomed Eng. 2002;30:392-401.

21. Repetto R, Stocchino A, Cafferata C. Experimental investigation of vitreous humour motion within a human eye model. Phys Med Biol. 2005;50:4729-4743.

22. Kumar S, Acharya S, Beuerman R. Deposition of particles on ocular tissues and formation of krukenberg spindle, hyphema, and hypopyon. J Biomed Eng. 2007;129:174-186.

23. Abouali $\mathrm{O}$, Modareszadeh A, Ghaffarieh A, Tu J. Investigation of saccadic eye movement effects on the fluid dynamic in the anterior chamber. J Biomech Eng. 2012;134:021002.

24. Kaji Y, Oshika T, Usui T, Sakakibara J. Effect of shear stress on attachment of corneal endothelial cells in association with corneal endothelial cell loss after laser iridotomy. Cornea. 2005;24:S55-S58. 